\title{
Use of a circular fixator construct to facilitate closed reduction and percutaneous stabilization of a distal femoral physeal fracture in a dog
}

\author{
Matthew A. Lazarus, Daniel D. Lewis*, Matthew D. Johnson and Erin G. Porter \\ Department of Small Animal Clinical Sciences, College of Veterinary Medicine, University of Florida, Gainesville, \\ FL 32610, USA
}

\begin{abstract}
Background: Fractures of the distal femoral physis are the most common physeal fracture sustained by skeletally immature dogs. Reduction and stabilization of these fractures can sometimes be achieved through closed reduction, primarily in fractures that are nominally displaced. Circular external fixator constructs have been used to assist in indirect, closed reduction of fractures at other anatomic locations in dogs and this report describes application of this method to reduce a displaced Salter-Harris type II fracture of the distal femur in a 1-year-old dog.

Case Description: A 1-year-old female spayed Akita was referred for treatment of a Salter-Harris type II fracture of the right distal femur. The epiphyseal segment was laterally and slightly caudally displaced. Multiple attempts to manually reduce the fracture during surgery were unsuccessful, so a two-ring circular external fixator construct was applied to facilitate distraction and reduction. The construct was applied by placing a medial-to-lateral Kirschner wire in both the mid-femoral diaphysis and in the distal femoral epiphysis. Distraction of the construct provided sufficient separation of the fracture segments to facilitate near anatomic reduction. The fracture was stabilized with two percutaneously placed Steinmann pins placed in Rush fashion. Radiographic union was confirmed 5 weeks after surgery. The dog was not lame and was bearing more weight on the right pelvic limb, as assessed using force plate analysis, 9 months following surgery. Goniometric measurements of stifle range of motion and thigh muscle circumference were similar between the pelvic limbs.

Conclusion: Application of a two-ring circular construct would appear to be useful to facilitate closed reduction and percutaneous stabilization of distal femoral physeal fractures.
\end{abstract}

Keywords: Circular fixator, Closed reduction, Dogs, Femur, Salter-Harris fracture.

\section{Introduction}

Fractures of the distal femoral physis are the most common physeal fracture and account for $60 \%$ of all distal femoral fractures in dogs (Grauer et al., 1981; Marretta and Schrader, 1983). These fractures have traditionally been stabilized via open reduction (Guiot and Déjardin, 2018), but some distal femoral physeal fractures are amenable to closed reduction (Kim et al., 2012; Boekhout-Ta et al., 2017). Addressing distal femoral physeal fractures via closed reduction and percutaneous pin placement may be advantageous by reducing iatrogenic trauma, mitigating post-operative pain, and accelerating fracture healing (Özsoy and Altunatmaz, 2003; von Laer, 2004; Kim et al., 2012; Boekhout-Ta et al., 2017). Obtaining acceptable closed reduction of fractures that are markedly displaced, however, is difficult (Kim et al., 2012). Intraoperative application of a two-ring circular external skeletal fixator construct has been described to facilitate indirect reduction and alignment of antebrachial and crural fractures in dogs undergoing minimally invasive plate osteosynthesis (Baroncelli et al., 2012; Pozzi et al., 2012, 2013). This report describes the use of a circular construct to aid in closed reduction prior to fluoroscopic-assisted percutaneous pinning for stabilization of a distal femoral physeal fracture in a dog.

\section{Case Details}

A $28 \mathrm{~kg}, 1$-year-old female spayed Akita presented for evaluation and treatment of a right distal femur fracture. The dog sustained the fracture after entangling the limb while jumping off a bed. The dog was evaluated by the primary veterinarian and radiographs were obtained which revealed a fracture of the right distal femur. A splint was applied to the fractured limb and the dog was referred.

On initial evaluation, the dog was bright, alert, and responsive, but placed limited weight on the splinted right pelvic limb. The dog was sedated and the splint was removed. The right stifle was swollen and there was palpable instability and crepitus elicited on manipulation of the right distal femur. Radiographs of the right femur were obtained. There was a sharply margined fracture that extended through the right distal femoral physis which included a small angular portion of the caudomedial aspect of the distal femoral metaphysis (Salter-Harris type II injury). The distal fracture segment was laterally and slightly caudally displaced. The soft tissues along the medial aspect of 
the distal femur were moderately thickened (Fig. 1A and B). Surgery was scheduled for the following day. The dog was anesthetized and the right pelvic limb was clipped and aseptically prepared for surgery. The dog was positioned in dorsal recumbency and several attempts were made to manually reduce the fracture in closed fashion; traction was applied to the distal limb as the femur was angulated through the fracture site and digital pressure was applied to the lateral femoral condyle, but the epiphyseal segment could not be reduced. A decision was made to transiently apply a circular fixator (IMEX Veterinary, Inc., Longview, TX) to facilitate closed reduction of the fracture (Pozzi et al., 2013; Gilbert et al., 2017; Hudson et al., 2020). A medial-to-lateral $1.6 \mathrm{~mm}$ Kirschner wire was placed through the distal femoral epiphysis. The wire was placed perpendicular to the frontal plane anatomic axis of the distal epiphysis. A second lateral-to-medial 1.6 $\mathrm{mm}$ Kirschner wire was inserted in the mid-diaphysis of the femur, perpendicular to the proximal femoral frontal plane anatomic axis. A C-arm (Fluoroscan InSight Mini-C-arm: Hologic, Diagnostic Health Care Systems, Raleigh, NC) was used to confirm appropriate placement of the fixation wires. The circular fixator consisted of two $118 \mathrm{~mm}$ diameter stretch rings and three $6 \mathrm{~mm}$ diameter threaded rods which were used to articulate the rings. The rods were $150 \mathrm{~mm}$ in length and were positioned lateral, cranial, and medial with the open section of the stretch rings positioned caudally. The Kirschner wires were attached to their respective rings using wire fixation bolts and nuts (Fig. 2). Initially manual traction was applied by grasping and physically separating the two rings. When soft tissue tension limited further manual distraction, the nuts located on the interior surface of the proximal ring were advanced along the rods until these nuts contacted the ring (Fig. 3A and B). Distraction of the fracture was assessed fluoroscopically and was not considered adequate to allow reduction. Additional distraction was performed by using a wrench to advance the nuts further proximally on the rods, increasing the distance between the two rings. When the fracture appeared to be adequately distracted to separate the epiphysis from the metaphysis, partial reduction was obtained by applying digital pressure to the medial femoral condyle. A large point-to-point forceps (Synthes, West Chester, PA) was applied through stab incisions to improve reduction in the frontal plane with the points of the forceps applied to the lateral surface of the epiphysis and the medial surface of the metaphysis (Fig. 3C). Reduction was improved, but still not acceptable, so the threaded rods were removed which allowed the distal stretch ring securing the epiphysis to be used as a traction bow (Peirone et al., 2020) to apply a varus stress to the epiphysis. A varus stress was also applied to the tibia eliciting a palpable and an auditable click. Acceptable reduction was confirmed via fluoroscopy (Fig. 3D). The reduction was stable and maintained without re-application of the connecting rods. Two 2.0 $\mathrm{mm}$ Steinmann pins were inserted in the epiphysis via individual $1.5 \mathrm{~cm}$ incisions with the stifle positioned in flexion and a proximally directed force was applied to the tibia (Fig. 3E). One pin was inserted in the lateral condyle at the level of the tendon of origin of the long digital extensor muscle and the second pin was inserted in a corresponding location on the medial femoral condyle. The pins were inserted in Rush fashion using the oscillating mode on the drill (Model\# OR6-6-1000, Advanced Mechanical Technology Inc., Newton, MA), until contact of two cortices was obtained. Fluoroscopic imaging confirmed appropriate pin placement. The pins were trimmed and countersunk using a pin setter (Fig. $3 \mathrm{~F})$. Closure was routine.

Radiographic images of the right femur were obtained immediately following surgery. The reduction was near anatomic as there was mild widening of the caudomedial aspects of the physis. The Steinmann pins were placed appropriately in Rush fashion. There was a mild increase in soft tissue opacity in the stifle joint, compared to preoperative radiographs, likely due to hemorrhage, and the soft tissues surrounding the distal femur remained thickened (Fig. 1C and D). The dog was intermittently placing weight on the right pelvic limb when discharged the day following surgery. The owners were instructed to keep the dog confined to a crate or small room when unattended and restrict the dog's outdoor activities to short walks on a leash for the first 4 weeks following surgery. Performing passive range of motion exercises on the right stifle was also recommended.

The dog was re-evaluated 5 weeks following surgery at which time the owner felt the dog was comfortable and using the right pelvic limb normally. On the orthopedic examination, the dog did not have a discernable lameness. Range of motion in the right stifle was only slightly reduced and pain was not elicited on flexion or extension. Radiographically, reduction was unchanged and the fracture margins were ill-defined, indicative of early healing. Along the medial aspect of the distal femoral metaphysis, there was a triangular region of smooth osseous proliferation ascribed to periosteal disruption. The Steinman pin in the lateral femoral condyle extended $1 \mathrm{~mm}$ distal to the margin of the subchondral bone, but the position of the implants appeared otherwise unchanged. Joint effusion and soft tissue swelling had resolved (Fig. 1E and F). The owners were advised to continue activity restriction for another 2 weeks before the dog could resume normal activities.

The dog was re-evaluated 9 months after surgery. The owner stated that the dog had resumed all normal activities without appreciable lameness and was very satisfied with the surgical outcome. On examination, the dog was not obviously lame. Force plate analysis was performed and peak vertical force was $12 \%$ less in the left pelvic limb than the right pelvic limb. There 

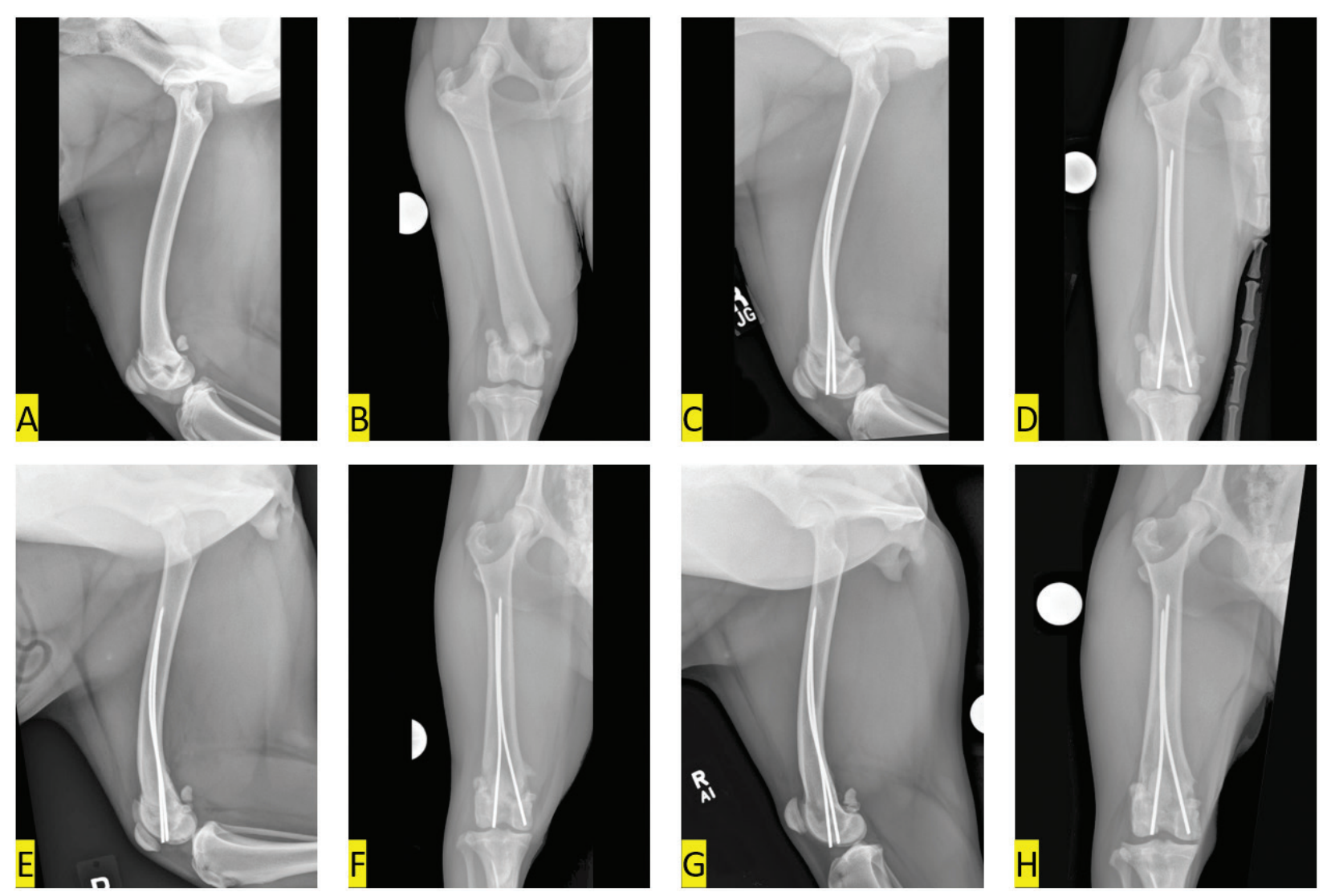

Fig. 1. Mediolateral (A, C, E, G) and caudocranial (B, D, F, H) radiographic images of the right femur. (A, B) pre-operative images show a laterally and slightly caudally displaced Salter-Harris type II fracture of the distal femur; (C, D) immediate post-operative images show the distal epiphysis has been nearly anatomically reduced. Two Steinmann pins have been placed in Rush fashion to stabilize the reduced fracture; (E, F) 5-weeks post-operative images of the right femur show the fracture remains reduced and aligned. The fracture margins are ill-defined, indicative of early healing. The Steinman pin in the lateral femoral condyle extends 1 $\mathrm{mm}$ distal to the lateral femoral condylar margin, but position of the implants seems otherwise unchanged; $(\mathrm{G}, \mathrm{H})$ 9-months postoperative images of the right femur show complete healing of the fracture with nominal stifle osteoarthrosis.

was full range of motion of the right stifle and no pain was elicited on flexion or extension. Goniometry was performed under sedation and flexion of the right stifle measured $30^{\circ}$, compared to $28^{\circ}$ in the left stifle. Extension of the right stifle measured $168^{\circ}$, compared to $166^{\circ}$ in the left stifle. Thigh circumference measured $39.6 \mathrm{~cm}$ and $39.2 \mathrm{~cm}$ in the right and left pelvic limbs, respectively. Radiographs confirmed complete union of the previously described fracture with minimal stifle osteoarthrosis and unchanged implants (Fig. 1G and H).

\section{Discussion}

Successful percutaneous pinning of distal femoral physeal fractures has been described, but has primarily been advocated for minimally displaced fractures (Pozzi and Thieman, 2011; Kim et al., 2012; BoekhoutTa et al., 2017). If the epiphysis is caudoproximally displaced (Tomlinson, 2005), but is still engaged with the metaphysis, the fracture may be amenable to closed reduction by applying traction (Pozzi and Thieman,
2011; Kim et al., 2012; Boekhout-Ta et al., 2017). Final reduction is often achieved by positioning the stifle in flexion and using the tibial plateau to push the femoral condyles cranially while applying a caudally directed pressure to the proximal femoral segment (Olmstead, 1984; Schrader, 1994). The epiphysis in the dog in the current report had displaced laterally and the medial metaphyseal pegs no longer engaged the pyramidal grooves of the epiphysis. Attempts to manually reposition the metaphyseal pegs in the appropriate corresponding pyramidal grooves, using combinations of traction applied to the tibia with angulation were unsuccessful. We were reluctant to continue to attempt manual closed reduction, as excessive forceful manipulations can cause additional physeal damage (Salter and Harris, 1963; Sponseller and Stanitski, 2001; Tomlinson, 2005). Iatrogenic trauma can be mitigated if manipulations are primarily traction (Sponseller and Stanitski, 2001; Kim et al., 2012), so the circular construct was applied to distract the 


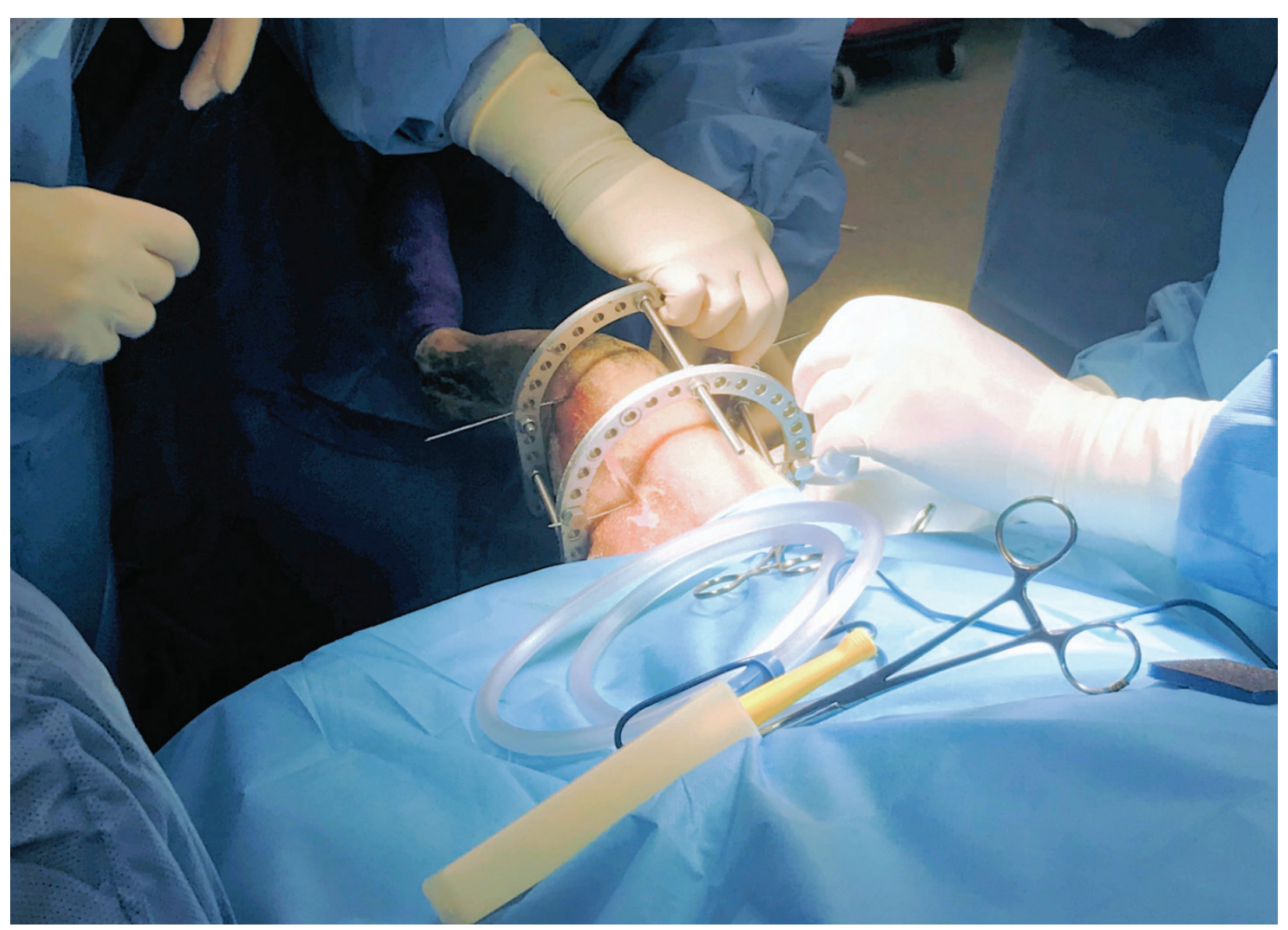

Fig. 2. Intra-operative image of the two-ring circular construct applied to distract the fracture segments.

femur through the fracture. Separation of the femoral segments allowed the metaphyseal pegs to disengage from the epiphysis. Rotation of the nuts positioned on the interior surface of the proximal ring on the threaded connecting rods utilized the leverage of the incline plane (Spinney, 1922) to separate the rings to overcome the tension asserted on the epiphysis by the quadriceps and hamstring muscles.

Transient application of circular constructs has been used most commonly to facilitate minimally invasive plate osteosynthesis of antebrachial and crural fractures (Baroncelli et al., 2012; Pozzi et al., 2012, 2013; Hudson et al., 2020), but fixators can also be used to facilitate closed reduction and alignment of humeral and femur fractures (Peirone et al., 2020). Temporary intra-operative application of a trans-articular circular construct has been reported to successfully reduce a markedly displaced SalterHarris type II fracture of the proximal tibia in a dog (Johnson et al., 2017). The authors of the current case report had previously attempted closed reduction of a type II Salter-Harris fracture of the distal femur using trans-articular application of a circular construct, but angulation of the stifle occurred during distraction and prevented successful indirect reduction. By placing one Kirschner wire in the femoral diaphysis and the other wire in the epiphysis, we were able to distract the fracture sufficiently to disengage the epiphysis and the metaphysis and realign the femur. The wire placed in the epiphysis in the dog in the current report was inserted slightly oblique to frontal plane anatomic axes which may have limited our ability to fully reduce the distracted epiphysis, even when point-to-point reduction forceps were applied, while the fixator was in place. Placing both fixation wires in the mediolateral plane allows for frontal plane translation of the secured bone segments following distraction (Anderson et al., 2003; Lewis and Farese, 2014; Hudson et al., 2020), but overcoming the force of the regional musculature caused substantial bowing of the fixation wires as the construct was distracted which also likely impeded our ability to effectively translate the engaged femoral segments and obtain complete anatomic reduction. We removed the connecting rods and utilized the distal ring as a traction bow (Peirone et al., 2020) to manipulate the epiphyseal segment to improve reduction. The tibia was abducted to obtain optimal frontal plane alignment (Bone, 1994) while we simultaneously flexed the stifle 

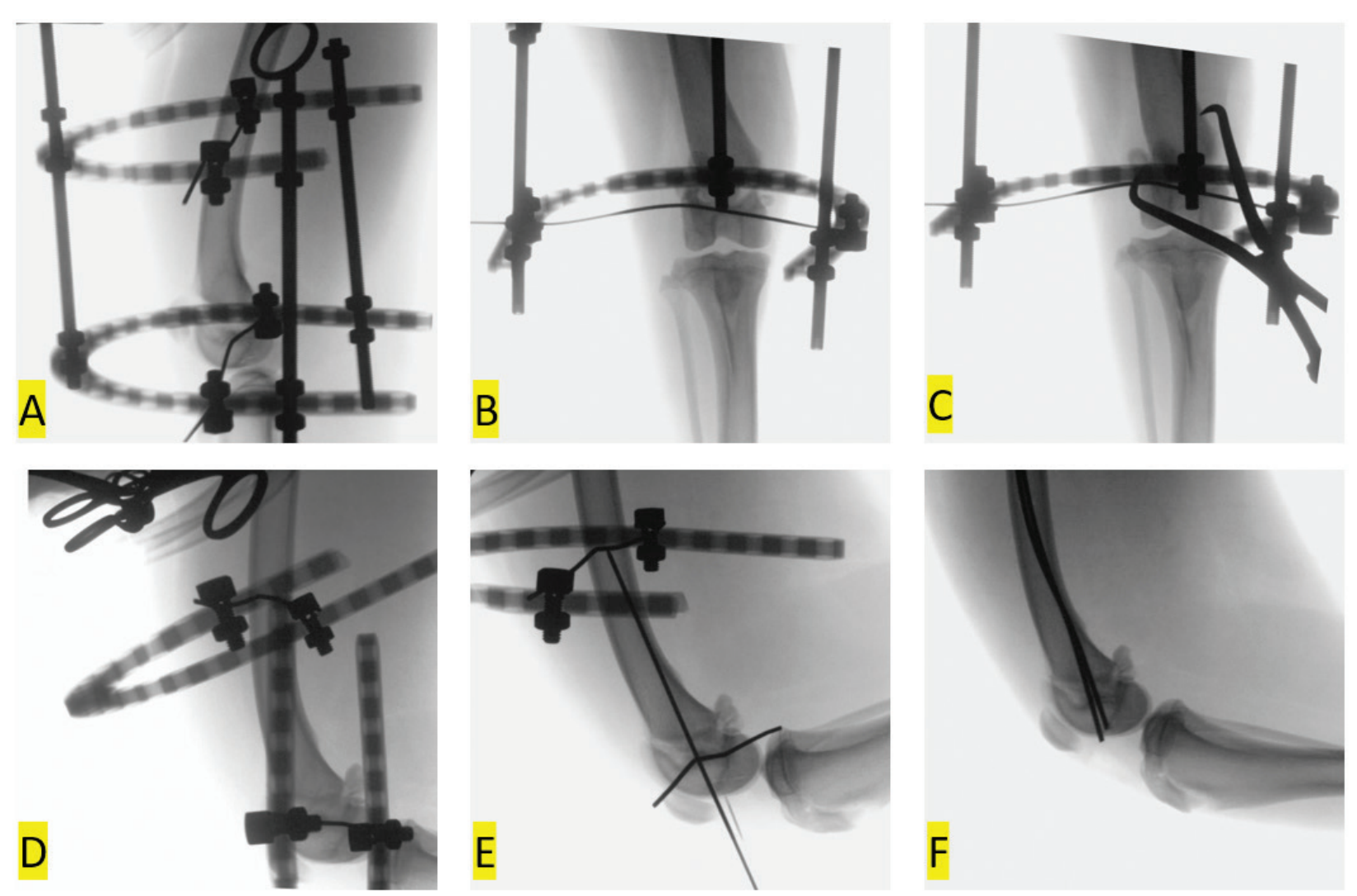

Fig. 3. Intra-operative fluoroscopic images of indirect closed fracture reduction and fluoroscopic assisted percutaneous pinning. The fixator was applied to distract the femur through the fracture site (A, B). A point-to-point reduction forceps was placed in an attempt to obtain anatomic reduction, but reduction was still suboptimal $(\mathrm{C})$. The connecting rods were removed and the ring secured to the distal fixation wire and used as a traction bow to apply a varus through the fracture as the stifle was flexed and a proximal force was applied to the tibia (D). A needle was used to locate an insertion site on the lateral femoral condyle and the first of two Steinmann pins were placed in Rush fashion (E). After placement the Steinmann pins were trimmed and counter sunk (F).

and applied a proximally directed force to the tibia to optimize sagittal reduction.

The use of intra-operative fluoroscopic imaging was integral to obtaining and confirming acceptable closed reduction and implant placement. Fluoroscopic assisted percutaneous pinning is a form of minimally invasive fracture repair involving closed reduction and placement of interfragmentary Kirschner wires or Steinmann pins inserted through small insertional incisions (Kim et al., 2012; Boekhout-Ta et al., 2017). We initially placed a hypodermic needle through the soft tissues cranial to the lateral femoral condyle to confirm, via fluoroscopy, the appropriate location for inserting our first Steinmann pin. Fluoroscopic assisted percutaneous pinning confers several advantageous benefits compared to traditional open techniques, including limited iatrogenic soft tissues trauma and less disruption to the peri-osseous circulation (Kreder et al., 2005; Boekhout-Ta et al., 2017).

We cut and then counter sunk the Steinmann pins through the pin insertional incisions which eliminated soft tissue irritation. The pin placed in the lateral femoral condyle was noted to protrude slightly beyond the subchondral bone on the 1- and 9-months postoperative radiographs. This pin did not appear to have migrated and there were no clinical or radiographic abnormalities suggesting that the pin was problematic. In a retrospective study assessing the clinical results of fluoroscopic-assisted percutaneous pinning of 42 physeal fractures in dogs and cats, Boekhout-Ta et al. (2017) reported elective pin removal was performed in $41 \%$ of cases. Management of the trimmed end of the pins was variable and was not described for individual cases, but surprisingly, the manner in which the end of the pins was managed was not found to be a significant predictor of pin removal or outcome (Boekhout-Ta et al., 2017). We would suggest that countersinking pins after the pins have been trimmed would cause less post-operative morbidity compared to leaving the pin protruding, even if bent, through the cartilage surface. Transient intra-operative application of the circular construct to distract the fracture segments facilitated successful indirect closed reduction of this dog's distal femur physeal fracture. Fluoroscopic-assisted 
percutaneous placement of Steinmann pins in Rush fashion resulted in a successful outcome. Closed reduction of fractures purportedly mitigates postoperative pain, leading to faster recoveries with decreased infection rates (Cook et al., 1999; Kreder et al., 2005; Boekhout-Ta et al., 2017). When the owner was contacted 2 days after discharge, the dog was already placing substantial weight on the right pelvic limb. The dog was not appreciably lame nor was discomfort elicited on manipulation of the right stifle 5 weeks following surgery. The owner of the dog was solicited to bring the dog back 9 months after surgery for a mid-term evaluation (Cook et al., 2010). The owner was very satisfied with the outcome and there was no lameness or discomfort noted on examination. Thigh circumference as well as flexion and extension of the stifles was comparable between pelvic limbs and, based on the results of the force plate analysis, the dog was surprisingly bearing more weight on the previously operated, right pelvic limb compared to the unoperated, left pelvic limb. The post-operative functional results in the dog in the current report corroborate results reported in a study of human patients with articular distal radius fractures managed with indirect reduction and percutaneous fixation who had an earlier return to function compared to patients managed with open reduction and internal fixation: patients treated by indirect reduction and percutaneous fixation also had superior long-term functional outcomes (Kreder et al., 2005).

\section{Conflict of interest}

The corresponding author teaches continuing education courses for IMEX Veterinary Inc. The other authors have no conflict of interest to declare relative to the work presented here.

References

Anderson, G.M., Lewis, D.D., Radasch, R.M., Marcellin-Little, D.J., Degna, M.T. and Cross, A.R. 2003. Circular external skeletal fixation stabilization of antebrachial and crural fractures in 25 dogs. J. Am. Anim. Hosp. Assoc. 39, 479-498.

Baroncelli, A.B., Peirone, B., Winter, M.D., Reese, D.J. and Pozzi, A. 2012. Retrospective comparison between minimally invasive plate osteosynthesis and open plating for tibial fractures in dogs. Vet. Comp. Orthop. Traumatol. 5, 410-417.

Boekhout-Ta, C.L., Kim, S.E., Cross, A.R., Pozzi, A. and Evans, R. 2017. Closed reduction and fluoroscopic assisted percutaneous pinning of 42 physeal fractures in 37 dogs and 4 cats. Vet. Surg. 46, 103-110.

Bone, L.B. 1994. Indirect fracture reduction: a technique for minimizing surgical trauma. J. Am. Acad. Orthop. Surg. 2, 247-254.

Cook, J.L., Evans, R., Conzemius, M.G., Lascelles, B.D., McIlwraith, C.W., Pozzi, A., Clegg, P., Innes, J., Schulz, K., Houlton, J., Fortier, L., Cross,
A.R., Hayashi, K., Kapatkin, A., Brown, D.C. and Stewart, A. 2010. Proposed definitions and criteria for reporting time frame, outcome, and complications for clinical orthopedic studies in veterinary medicine. Vet. Surg. 39(8), 905-908.

Cook, J.L., Tomlinson, J.L. and Reed, A.L. 1999. Fluoroscopically guided closed reduction and internal fixation of fractures of the lateral portion of the humeral condyle: prospective clinical study of the technique and results in ten dogs. Vet. Surg. 28, 315-321.

Gilbert, E.D., Lewis, D.D., Townsend, S. and Kim, S.E. 2017. Comparison of two external fixator systems for fracture reduction during minimally invasive plate osteosynthesis in simulated antebrachial fractures. Vet. Surg. 46, 971-980.

Grauer, G.F., Banks, W.J., Ellison, G.W. and Rouse, G.P. 1981. Incidence and mechanisms of distal femoral physeal fractures in the dog and cat. J. Am. Anim. Hosp. Assoc. 17, 579-586.

Guiot, L.P. and Déjardin, L.M. 2018. Fractures of the femur. In Veterinary surgery small animal, Eds., Johnston, S.A. and Tobias, K.M. St. Louis, MO: Elsevier, pp: 1019-1064.

Hudson, C.C., Lewis, D.D. and Pozzi, A. 2020. Minimally invasive plate osteosynthesis radius and ulna. Vet. Clin. North Am. Small Anim. Pract. 50, 135-153.

Johnson, M.D., Lewis, D.D. and Winter, M.D. 2017. Intraoperative use of a transarticular circular fixator construct to facilitate reduction and stabilization of a proximal tibial physeal fracture in a dog. Aust. Vet. J. 95, 161-166.

Kim, S.E., Hudson, C.C. and Pozzi, A. 2012. Percutaneous pinning for fracture repair in dogs and cats. Vet. Clin. North Am. Small Anim. Pract. 42, 963-974.

Kreder, H.J., Hanel, D.P., Agel, J., McKee, M., Schemitsch, E.H., Trumble, T.E. and Stephen, D. 2005. Indirect reduction and percutaneous fixation versus open reduction and internal fixation for displaced intra-articular fractures of the distal radius: a randomised, controlled trial. J. Bone Joint Surg. Br. 87(6), 829-836.

Lewis, D.D. and Farese, J.P. 2014. Circular external skeletal fixation. In: Current techniques in small animal surgery. Eds., Bojrab, M.J., Waldron, D. and Toombs, J.P. Jackson, WY: Teton NewMedia, pp: 828-843.

Marretta, S.M. and Schrader, S.C. 1983. Physeal injuries in the dog: a review of 135 cases. J. Am. Vet. Med. Assoc. 182, 708-710.

Olmstead, M.L. 1984. Fractures of the femur. In: Manual of internal fixation in small animals. Eds., Brinker, W.E., Hohn, R.B., Prieur. New York, NY: Springer-Verlag, pp: 165-175.

Özsoy, S. and Altunatmaz, K. 2003. Treatment of extremity fractures in dogs using external fixators 
with closed reduction and limited open approach. Vet. Med. Czech. 48, 133-140.

Peirone, B., Rovesti, G.L., Baroncelli, A.B. and Piras, L.A. 2020. Minimally invasive plate osteosynthesis fracture reduction techniques in small animals. Vet. Clin. North Am. Small Anim. Pract. 50, 23-47.

Pozzi, A. and Thieman, K.M. 2011. Percutaneous pinning of growth plate fractures in dogs. Vet. Comp. Orthop. Traumatol. 4, A13-A14.

Pozzi, A., Hudson, C.C., Gauthier, M. and Lewis, D.D. 2013. Retrospective comparison of minimally invasive plate osteosynthesis and open reduction and internal fixation of radius-ulna fractures in dogs. Vet. Surg. 42, 19-27.

Pozzi, A., Risselada, M. and Winter, M.D. 2012. Ultrasonographic and radiographic assessment of fracture healing after minimally invasive plate osteosynthesis and open reduction and internal fixation of radius-ulna fractures in dogs. J. Am. Vet. Med. Assoc. 241, 744-753.

Salter, R.B. and Harris, W.R. 1963. Injuries involving the epiphyseal plate. J. Bone Joint Surg. Am. 277, $7-71$.
Schrader, S. 1994. Fractures of the femur. In: The cat: diseases and clinical management. Ed., Scherding, R. New York, NY: Churchill Livingstone Inc., pp: 1668-1678.

Spinney, L.B. 1922. The simple machines. In: A textbook of physics. Ed., Spinney, L.B. New York, NY: The Macmillan Company, pp: 87-97.

Sponseller, P.D. and Stanitski, C.L. 2001. Fractures and dislocations about the knee. In: Rockwood and Wilkins' fractures in children. Eds., Beaty, J.H. and Kasser, J.R. Philadelphia, PA: Lippincott Williams \& Wilkins, pp: 1433-1576.

Tomlinson, J. 2005. Fractures of the distal femur. In: AO principles of fracture management in the dog and cat. Eds., Johnson, A.L., Houlton, J.E.F. and Vannini, R. Davos, Switzerland: AO Publishing, pp: 297-303.

von Laer, L. 2004. General observations on treatment. In: Pediatric fractures and dislocations. Ed., von Laer, L. Stuttgart, Germany: Thieme, pp: 69-77. 